\title{
Current Situation and Progress in Treatment of Ascites by Reinfusion
}

\author{
Kunpeng Tian (Corresponding author) \& Yajing Li \\ Institute of Biological \& Chemical Engineering \\ Tianjin Polytechnic University, Tianjin 300160, China \\ E-mail: dongwuxue408@163.com
}

\begin{abstract}
Ascites, an abnormal accumulation of fluid in the abdomen, is often associated with liver cirrhosis, peritoneal tuberculosis, peritoneal metastatic carcinoma and primary peritoneal tumor. It not only affects the quality of life, but also shortens the survival time. Ascites is usually treated with the conventional therapy, diuretic drugs, abdominal paracentesis, peritoneovenous shunt, volume expansion therapy, liver transplantation and etc. Treatment of ascites by reinfusion is easy to handle, cheap, effective and easily accepted by sufferers. Therefore, this paper introduces the current situation and progress in the treatment of ascites by reinfusion.
\end{abstract}

Keywords: Ultrafiltration of ascitic fluid, Ascites reinfusion

\section{Introduction}

Ascites is an abnormal accumulation of fluid in the abdomen. Small amounts of fluid in the abdomen $(<100 \mathrm{~mL})$ can lubricate the internal organs, such as stomach and intestinal tract. However, some patients develop excessive free liquid (common) or localized liquid (relatively uncommon) in the abdomen, which is usually more than $100 \mathrm{~mL}$, sometimes reaches hundreds or even ten thousands of millilitres (Xie, Pengyan, 2006, p. 2-3). The reasons that cause ascites are complicated, but liver cirrhosis, tumor and tuberculosis are responsible for about $50 \%$ of all instances of ascities (Xu, Dayi, 2007). Laboratory analysis of fluid extracted by abdominal paracentesis is a preferred diagnosis technology, which can determine whether the fluid is an exudate or a transudate, benign or malign. With the development of biochemical technology, the biochemical indexes and radiography of fluid play important roles in diagnosis of ascites, and help identify the cause of the accumulation. However, ascites of different natures have many overlapped indexes. Therefore, ascites can not be accurately characterized by single index, and combined detection of several indexes may increase the accuracy of diagnosis (Ni, Runzhou et al, 2007, p. 110-113).

Ascites is usually treated with the conventional therapy, diuretic drugs, abdominal paracentesis, peritoneovenous shunt, volume expansion therapy, liver transplantation and etc. However, different from paracentesis in which fluid is directly drained from a body cavity, and also different from albumin injection in which albumin functions to maintain the osmotic pressure in a normal range and keep fluid inside the blood vessels, treatment of ascites by reinfusion can help eliminate excess fluid and expand volume at the same time, meanwhile, it is easy to handle, cheap, effective and easily accepted by sufferers. Ascites reinfusion includes intravenous reinfusion of ascites, abdominal reinfusion of concentrated ascites and subcutaneous reinfusion of concentrated ascites.

\section{Intravenous reinfusion of ascites}

\subsection{Peritoneovenous shunt (PV - shunt)}

Take Leveen peritoneovenous shunt as an example, the surgeon attaches a pressure-sensitive one-way valve to prevent backflow, and inserts a silicone tube that connects the peritoneal cavity to the jugular vein. PV-shunt may be performed to achieve the continuous emptying of ascitic fluid into the venous system, increase the renal blood flow and urinary output, inhibit renin and angiotonin, and relieve patients' pain, to whom urine-producing drugs are ineffective. However, it usually causes complications, such as DIC, peritonitis, hematosepsis, cardiac insufficiency and etc. (Wang, Jinke, 2000, p. 1277-1282). The severest defect thereof is that the silicone tube is easy to be blocked. To overcome this shortcoming, Zerros et al developed a Denver-type shunting tube that is effective to 48 instances of intractable ascites (Wang, Kangying et al, 2000, p. 113). However, this tube could still be blocked in one year, and caused many complications. 


\subsection{Direct intravenous reinfusion of ascites}

A direct intravenous reinfusion process refers to a procedure in which ascitic fluid extracted by abdominal paracentesis is directly reinfused into patient's venous system. It consists of single intravenous reinfusion process and intravenous reinfusion-diuretics combination process. This method can promote blood circulation, increase urinary output and $\mathrm{U}_{\mathrm{Na}}$ excretion, decrease ascitic fluid, and avoid loss of protein and electrolytes (Bai, Wenyuan et al, 2002, p. 159). After direct intravenous reinfusion of ascites, patient's body reveals the increase of creatinine removal rate, free water removal rate and albumin level, and decrease of plasma aldosterone. However, it may cause early adverse reactions. Li, Yuling et al (1999, p. 91) reported that reinfusion of intravenous medicine-contained ascites, followed by intravenous injection of $20 \mathrm{mg}$ furosemide, had good effects on 30 patients. Xu, Linshou et al (2001, p. 507-508) reported that direct intravenous reinfusion, followed by intake of Chinese herbs containing hard lump removers and spleen invigorator, not only had good effects on treatment, but also prevented new fluid accumulations, and its 3-month non-recurrent rate is up to $76.6 \%$.

\subsection{Intravenous reinfusion of concentrated ascites}

Part of water and micromolecular compounds are removed from ascitic fluid by ultrafiltration or dialysis, then the obtained concentrated ascites containing protein and other useful substances is infused back into the patient. Its action mechanisms are as following: (1) the remarkable decrease of ascitic fluid causes the decrease of intra-abdominal pressure, improvement of renal blood perfusion, as well as increase of glomerular filtration rate and excretion of water and $\mathrm{Na}^{+} ;(2)$ the protein in reinfused fluid can increase osmotic pressure of plasma colloid; (3) the increase of effective blood volume and renal blood flow stops the activity of renin-angiotonin-aldosterone system, and inhibits the excretion of aldosterone and antidiuretic hormone. Although avoiding loss of protein, intravenous reinfusion of concentrated ascites may sometimes cause hematosepsis, excessive volume load, disseminated intravascular coagulation and other complications. At first, ascites is drained from a body cavity, and then reinfused into the abdomen after extracorporeal concentration, however, in which the open sterile chamber is susceptible to bacteria contamination, and it is rather time-consuming to perform hemodialysis and reinfusion separately. Therefore, many new methods for ascites concentration have been reported recently. Xu, Linshou et al (2001, p. 507-508) studied the treatment of abdominal ascites by concentration and reinfusion with artificial kidney. He performed abdominal paracentesis with fistula needle at both sides of lower abdomen, drained the ascitic fluid into an artificial kidney to form a closed cyclic dialysis and ultrafiltration system of ascites, and reinfused the concentrated ascitic fluid into vein. This method can reinfuse the ascitic fluid into abdominal cavity and vein, and avoid the bacteria contamination from extracorporeal sterile chamber.

\section{Abdominal reinfusion of concentrated ascites}

Abdominal reinfusion of concentrated ascites has been a new method for treatment of different intractable ascites in recent years home and abroad. It is a simple, safe and reliable procedure in which the ascitic fluid is drained from abdominal cavity, and infused into abdominal cavity again after ultrafiltration concentration. This method avoids the loss of endogeneous protein, and increases the activity of opsonin in the remained fluid. Compared with intravenous reinfusion, this method reveals a comparative curative effect, but a higher safety, avoids the adverse effects and complications that intravenous reinfusion could cause, and broadens the treatment possibilities of intractable ascites, such as ascites caused by cancer, hemorrhagic disease, infection, cardiogenic disease and nephrogenic disease.

$\mathrm{Hu}$, Darong et al (2001, p. 89) reported that 311 patients with intractable ascites had been treated by the ultrafiltration concentration and abdominal reinfusion for 921 times, and the total effective rate was $69.7 \%$. Liu, Limin et al (2004, p. 63) treated 8 patients by abdominal reinfusion of concentrated ascites, and compared with other 6 patients treated by intravenous reinfusion of ascites. The result revealed that abdominal reinfusion of concentrated ascites was more effective in preventing patients from infection, and caused fewer complications than other methods. Zhang, Jinlong et al (2003, p. 294-295) treated 42 patients by abdominal reinfusion of concentrated ascites, followed by injection of 20240 $\mathrm{mg}$ dopamine, $402120 \mathrm{mg}$ furosemidum, as well as $1 \mathrm{~g}$ ceftriaxone sodium or $0.75 \mathrm{~g}$ cefuroxime into abdominal cavity. This method not only solved the issues of massive ascites and deficiency of effective circulating blood volume, but also prevented patients from infection and complications. Its total effective rate was up to $95 \%$.

As for the treatment of intractable ascites, abdominal reinfusion of concentrated ascites is a much safer, more effective, more time-saving and cheaper method, which solves some hard issues, and improves the patients' life quality. However, abdominal reinfusion of concentrated ascites still has some defects, such as fluid clotting, tube blockage, infection, as well as acute pneumonedema and bleeding of venae oesophageae resulted from rapid or massive reinfusion of concentrated ascites (Xu, Chengrun et al, 2005, p.176).

\section{Subcutaneous reinfusion of concentrated ascites}

Li, Haijian et al (2000, p. 388-389) reported that 17 patients had been treated by subcutaneous reinfusion of concentrated ascites. This treatment has a little effects on 8 patients, and obvious effects on 9 patients. Its curative effect is similar to that of intravenous reinfusion. No obvious complications were observed. 


\section{References}

Bai, Wenyuan \& Yao, Dongmei. (2002). Treatment of ascites caused by liver cirrhosis by interferon. Diagnosis and Treatment of Digestive Disease, 2(4), 159.

$\mathrm{Hu}$, Darong, Wang, Yi, Tian, Huiying, Wang, Chunni, Peng, Xiaojun \& Gong, Juan. (2001). Treatment of intractable ascites by ultrafiltration concentration and abdominal reinfusion: analysis on 921 cases of treatment. Chinese Hepatology, 6(4), 89.

Li, Haijian, Huang, Yongzhi \& Lu, Pingxuan. (2000). Clinic study on treatment of intractable ascites by concentration and abdominal reinfusion. Journal of Youjiang Medical College for Nationalities, 22(3), 388-389.

Li, Yuling \& Luee, Aiyun. (1999). Observation on curative effect of abdominal reinfusion of concentrated ascites on 30 patients with intractable ascites caused by liver cirrhosis. Henan Journal of Diagnosis and Treatment, $13(2), 91$.

Liu, Limin, Chi, Yanchun, Zhu, Dan, Yang, Xiaomei \& Sun, Lihong. (2004). Closed extracorporal concentration and reinfusion of ascites in cirrhosis of liver patients with intractable ascites. Chinese Journal of Hepatology, 12 (5), 63.

Ni, Runzhou \& Meng, Xianyong. (2007). Clinic significance of laboratory analysis on ascitic fluid. Medical Journal of Communications, 21(2), 110-113.

Wang, Jinke. (2000). Mechanism of ascites caused by liver cirrhosis and progress in its treatment. Guangxi Medical Journal, 22(6), 1277-1282.

Wang, Kangying, Yang, Haimin \& An, Changxue. (2000). Observation on curative effect of direct intravenous reinfusion combined with Chinese medicine on liver cirrhosis. Infections Disease Information, $13(3), 113$.

Xie, Pengyan. (2006). Basic conception on identification of ascitic fluid. Chinese Journal of Medicine, 41(9), 2-3.

$\mathrm{Xu}$, Chengrun \& Rao, Richun. (2005). Research progress in treatment of intractable ascites by ultrafiltration concentration and abdominal reinfusion. Journal of Clinical Hepatology, 8(3), 176.

$\mathrm{Xu}$, Dayi. (2007). Current situation and progress in treatment of ascites. The 19th National Conference on Digestive Disease.

Xu, Linshou, Pan, Ronghua, Zhang, Yanlin, Zhou, Changcheng, Jia, Yuxin \& Zhao, Baiying. (2001). Study on treatment of intractable ascites caused by liver cirrhosis by concentration and reinfusion with artificial kidney. Jiangsu Medical Journal, 27(7), 507-508.

Zhang, Jinlong, Huang, Zhigang \& Mo, Guozhong. (2003). Treatment of 42 patients with intractable ascites caused by liver cirrhosis by ultrafiltration concentration and reinfusion. Chinese Journal of Infectious Diseases, 21(4), $294-295$. 